\title{
Analogical Cognition: Applications in Epistemology and the Philosophy of Mind and Language
}

Theodore Bach*

Bowling Green State University Firelands College

\begin{abstract}
Analogical cognition refers to the ability to detect, process, and learn from relational similarities. The study of analogical and similarity cognition is widely considered one of the 'success stories' of cognitive science, exhibiting convergence across many disciplines on foundational questions. Given the centrality of analogy to mind and knowledge, it would benefit philosophers investigating topics in epistemology and the philosophies of mind and language to become familiar with empirical models of analogical cognition. The goal of this essay is to describe recent empirical work on analogical cognition as well as model applications to philosophical topics. Topics to be discussed include the epistemological distinction between implicit knowledge and explicit knowledge, the debate between empiricists and nativists, the frame problem, expertise, creativity and autism, cognitive architecture, and relational knowledge. Particular attention is given to Dedre Gentner and colleague's structure-mapping theory - the most developed and widely accepted model of analogical cognition.
\end{abstract}

\section{Introduction}

Reflecting on the science of analogical cognition back in 1983, Fodor remarked:

It is striking that, while everybody thinks that analogical reasoning is an important ingredient in all sorts of cognitive achievements that we prize, nobody knows anything about how it works; not even in the dim, in-a-glass-darkly sort of way in which there are some ideas about how confirmation works. (Fodor 1983: 107)

Much has changed in the past 30 years. At present, work in the area of analogical and similarity cognition is considered one of the 'success stories' of cognitive science (Forbus et al. 1998: 231). Whereas cognitive scientists from different disciplines can sometimes 'talk past' one another (neurobiological reductions of consciousness that fail to explicate the philosopher's notion of consciousness, to mention one example - see Chalmers 1995), the study of analogical cognition has been a locus of fruitful interdisciplinary collaboration. To illustrate: Artificial Intelligence researchers who computationally simulate analogical cognition implement processing assumptions developed by cognitive psychologists. These processing assumptions, commonly expressed in the semantic propositional networks codified in linguistics, trace back to philosophical, theoretical models of analogy (e.g., Hesse 1966). In addition to collaborating, researchers have converged on answers to foundational questions about the nature of analogical cognition (Holyoak and Hummel 2001: 161; Gentner and Kurtz 2006: 609).

Given these theoretical and empirical strides, it is surprising that philosophers - particularly those investigating mind, language, and knowledge - have not paid closer attention to the science of analogy. If analogical cognition is as central to human cognition as 
several researchers suggest (see Section 3.4., below), then this could be an expensive oversight. The goal of the present article is to sketch some recent developments in the science of analogical cognition with a special focus on how these developments relate to broader themes in the philosophy of mind, language, and knowledge.

The article is selective in two respects. First, several theories of analogical cognition are currently on offer, and while there is considerable overlap between these theories, the present article restricts its focus to Gentner and colleagues' structure-mapping theory - the most widely accepted and widely applied theoretical framework for analogical cognition. Second, given that structure-mapping theory is a highly developed and nuanced research program, and given also that the philosophies of mind, language, and knowledge cover a vast and eclectic research territory (to put it mildly), the applications sketched here represent only a fraction of those that may be of philosophical interest. Nonetheless, if the article is successful it should be suggestive of other ways that analogy can bear on philosophical explananda.

\section{Analogical Cognition}

We often judge two things as the same, or remark that 'this is like that'. Depending on the type of comparison, different axes of similarity will have different levels importance. For example, a comparison between the moon and Swiss cheese invokes the superficial - or attributional (Gentner 1983) - similarity white hole-filled surface. On the other hand, understanding that 'a battery is like a reservoir' requires picking out the relational similarity stores potential energy. Gentner and Markman (1997) propose a similarity space that captures the different forms of similarity cognition (Fig. 1).

We can explore this space with the term jail (Gentner and Markman 1997: 48): 'My job is a jail' is an analogy, 'the Zebra is like a jail' is a mere-appearance match, 'the jail is a prison' expresses a literal similarity, and 'jail is an integer' is anomalous. Note that metaphors are not a natural kind of similarity on this model. A metaphor might express an analogy - 'Love is a journey' - or a mere appearance - 'the moon is cheese' - or some blend. In this respect, grouping similarity statements into the superordinate category metaphor is analogous to grouping deposits of Jadeite and Nephrite into the class Jade.

Our focus here is analogy. Analogies are comparisons that invoke relational similarities. As such, 'good' analogies do not require compared items to be attributionally similar. When Pat Benatar sings that 'Love is a Battlefield', the fact that a battlefield but not love is composed of soil does not detract from the strength of the comparison. Analogical

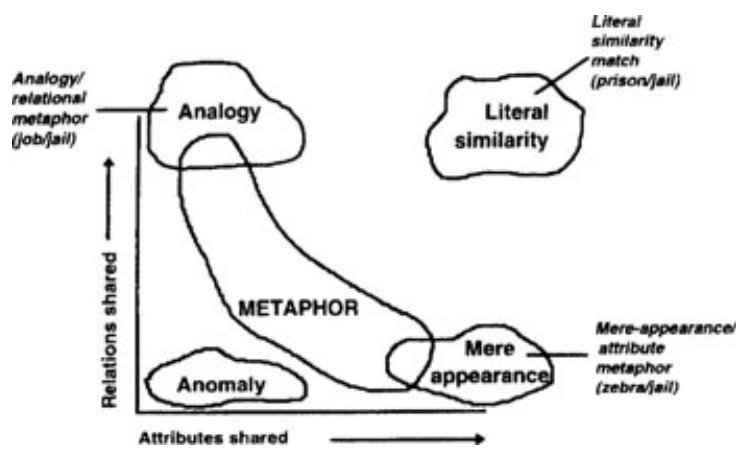

Fig. 1. Similarity space depicting types of similarity matches. (From Gentner and Markman 1997: 48) 
cognition is a type of similarity cognition that refers to the ability to detect, process, and learn from relational patterns. This ability is usefully broken down into several component processes (see Gentner and Medina 1998; Gentner and Colhoun 2010).

\section{I. RETRIEVAL}

In order to align a base representation and a target representation, as well as project inferences from the base to the target, one must first juxtapose (or activate) both representations in working memory. Analogical retrieval is the process through which a base representation is accessed from long-term memory. Retrieval may occur spontaneously, as when a current situation reminds one of a structurally similar episode, or it may occur through prompting, as when explicit instructions prompt the retrieval of a base representation. For example, when learning about the Rutherford atom one could be spontaneously reminded of the solar system, or one could be told that 'the atom is like the solar system.'

\subsection{ALIGNMENT, HighLIGHTING RELATIONS, AND INFERENCE PROJECTION}

All theories of analogy begin with a set of assumptions about knowledge representation. According to structure-mapping theory, representations explicitly encode attributes (predicates that take one argument such as red and grass), relations (predicates that take at least two arguments such as brother and above), and higher-order relations (predicates that take relations as arguments such as symmetrical and cause). A core process of analogical cognition involves aligning the elements of a base representation and a target representation and establishing correspondences. When aligning two representations, there is a drive to find the most structurally consistent mapping. This results in mappings that privilege relations and discard attributes. This bias is particularly clear in cases of 'cross-mapping', in which relational correspondences are pitted against and win out over literal similarities. For example, when aligning 'Salmon eat minnows' and 'Humans eat salmon', salmon is matched with humans (despite the identical match between salmon and salmon) because both occupy the relational role of eater in a structural system. There is strongest preference for higher-order relations, for example causal relations, which are relations that participate in a cohesive network of other relations.

A central insight of structure-mapping theory is that when learners consider one exemplar they are apt to focus on non-relational features, e.g., attributes, but when there are two exemplars and subjects compare them, the activity of alignment will highlight common relational structure. Metaphorically, when we align two representations a common relational structure will 'pop-out'. Gentner et al. (1997) call this 'the comparison as X-ray phenomena'. For example, aligning the atom representation and the solar-system representation can highlight the common relation: The planets/electrons REVOLVE AROUND the sun/nucleus. This relation is preserved because it is systematic - it is constrained by the higher order relation: The fact that the $X$ attracts $Y$ CAUSES $Y$ to revolve around $X$. On the other hand, the relation hotter than does not participate in an interconnected system of relations and is fortuitously discarded during the mapping process.

Systematic relations that are represented only in the base representation are excellent candidates for inferences to project to the target representation. For instance, someone with a good grasp of the mechanics of a solar system but not the Rutherford atom will likely carry over relations from the solar system representation to the atom representation. 


\subsection{EVALUATION}

The structural mappings and inferences that occur as a result of structural alignment are not guaranteed to correspond to mind-independent structural facts. Indeed, philosophers have often targeted this ampliative feature of analogy for criticism (e.g., the much maligned analogical argument for other minds). Evaluation involves invoking further criteria to assess the results of analogical mapping. In addition to evaluating objective correspondence, analogies can be evaluated on pragmatic grounds. (Holyoak and colleagues' pragmatic model of analogy incorporates a system's goals into the mapping phase.)

\subsection{SCHEMA ABSTRACTION}

An important type of learning through analogy occurs when learners abstract a relational schema as a result of comparison (see especially Gick and Holyoak 1983; Gentner 2005). Prior to structural alignment, a relational category may be understood contextually but not abstractly. Such a contextual grasp results in the conflation of relational categories with their object realizers. However, given the comparison-based relational focus discussed above, highlighted relational structure is made ripe for abstraction. Abstracted relational predicates and schemas can then serve as the basis for relational categories that extend more widely to object realizers. For example, by comparing the solar system and the atom, a learner could abstract a more general representation of a central force system.

\subsection{RE-REPRESENTATION AND PROGRESSIVE ALIGNMENT}

Re-representation occurs when a representation is changed during the comparison process in order to improve alignment. Imagine someone with an impoverished conception of the solar system - perhaps someone who understands the heat of the sun as causing the motion of the planets. During comparison, this individual may alter his or her representation of the solar system in order to maximize structural alignment.

Progressive alignment describes the process through which learners start by making simple comparisons supported by attribute similarities (which are easy for beginners), from which they abstract a bit of relational knowledge, and then on the basis of this knowledge are able to make comparisons less supported by perceptual/superficial similarities, and so on. As this process repeats, learners 'wear away' (Kuehne et al. 2000) the importance of attributes in their representation of categories. Importantly, learning through progressive alignment is possible because relational focus obtains even when the compared items are literally similar. Consider a learner with little grasp of what a central force system is, and for this reason cannot align, or detect correspondences between, a solar system and an atom. On the other hand, given the superficial overlap of two distinct solar systems, this learner is able to match one solar system with the other. The relational focus that occurs through this comparison will promote the salience of the relational features of the solar-systems while demoting the importance of the very attributes that encouraged the comparison. If relational structure is abstracted as a result, then this small learning achievement will permit more distant alignments, which can lead to greater relational abstraction, and so on.

A great deal of experimental work supports the above claims (see Gentner and Markman 1997 and Gentner and Colhoun 2010 for reviews). The processing principles of structural alignment, retrieval, and progressive alignment have also been computationally modeled with considerable success by, respectively, the Structure-Mapping-Engine 
(SME), MAC/FAC, and SEQL (see Gentner and Forbus 2011 for a recent review of computational models of analogy). Besides providing computational meaning to the spatial metaphor of structural 'alignment', these simulations demonstrate that a real-time learning mechanism can arrive at a deep, structural mapping without any prior programming for the target structure.

Traditional philosophical interest in analogy has tended to focus on the processes of relational mapping and inference projection. In the following section I suggest how other analogical processes, and in particular schema abstraction, re-representation, and progressive alignment, are or philosophical interest.

\section{Applications to Epistemology, Philosophy of Mind, and Philosophy of Language}

For the remainder of the article I sketch ways in which the science of analogical cognition can shed light on philosophical problems, particularly those that are salient in epistemology and the philosophies of mind and language. Specific topics to be discussed include: the distinction between implicit and explicit knowledge, the debate between nativists and empiricists (and relatedly, the debate between modularity theorists and theory-theorists), the frame problem, expertise, creativity and autism, cognitive architecture, and relational knowledge.

\section{I. IMPLICIT AND EXPLICIT KNOWLEDGE}

Researchers across several disciplines have interpreted the distinction between implicit and explicit knowledge in a variety of ways (see Dienes and Perner 1999; Davies 2001). A common interpretation of implicit knowledge invokes the use of a 'tacit theory'. Put roughly, a tacit theory, for example theory-of-mind, is any internally represented knowledge structure (Stich and Nichols 1992) that is not readily accessible to consciousness. In contrast, explicit theories are knowledge structures that are consciously accessible and available for verbal report. The vigorous debates between simulation-theorists and theory-theorists provide evidence for the difficulty involved in formulating this distinction precisely (see especially Davies and Stone 2001).

Gentner's discussion of comparison-based abstraction and progressive alignment suggests a distinct construal of the distinction between implicit and explicit knowledge. Formally, we can say that implicit knowledge is relational knowledge that is embedded in a particular context or conflated with a particular argument value. In contrast, explicit knowledge encodes a relation as multiply realizable by objects. Putting this difference in representational terms: implicit knowledge involves possession of a relational predicate that can take only one, or a set of superficially similar, argument values, while explicit knowledge involves possession of a relational predicate that can be matched across superficially different argument values. To demonstrate, a 3 year old who grasps that two people can use a telephone to communicate with one another, but who embeds this relational knowledge in a particular exemplar (i.e., a particular phone, a particular set of adults, or a phone of a particular color), has implicit rather than explicit relational knowledge of telecommunication. On the other hand, a 5 year old who grasps that Bluetooth headsets are used for telecommunication, and who possesses a representational symbol for telecommunication that can accept novel arguments, has explicit knowledge of the relation of telecommunication. (According to certain metaphysical functionalists, identitytheorists commit a sophisticated version of the child's error when they identify the relational category mental state with one of its object-level realizers, namely, brain states.) 
Progressive alignment through structural-comparison is then a learning mechanism that converts implicit knowledge into explicit knowledge. Prior to the relational shift in a domain (Gentner et al. 1995), children have only an implicit grasp of relations in that domain. This is because children conflate relations with specific argument values. After the shift, children have made explicit the relations that are the essential property of a relational category; they no longer conflate the relation with particular object-level realizers. Importantly, this version of the distinction between implicit and explicit knowledge is easily confused with the distinction between tacit and salient knowledge as described above. While the two distinctions can correspond (implicit knowledge that is tacit; explicit knowledge that is salient) they often come apart. Philosophers and psychologists researching and applying these concepts would benefit by noting the difference between these distinctions and keeping them separate.

\subsection{EMPIRICISM, NATIVISM, AND CONCEPTUAL LEARNING}

The labels 'nativism' and 'empiricism' each collect a variety of doctrines, and debates between nativists and empiricists take many forms. Here I focus on the issue of whether, and to what extent, conceptual representations - scripts, concepts, schemas, theories - are the product of domain-specific faculties (perhaps innately specified modules) or the product of domain-general learning mechanisms. This is a discussion in 'descriptive epistemology' (Goldman), but if one supplements it with a claim about the truth-tracking properties of concept-forming mechanisms - a claim supported in many naturalistic epistemologies - then one can draw normative epistemological conclusions as well.

We can think of domain-specific mechanisms as specialized for a specific type of content. For example, nativists posit mechanisms that have processing rules and/or innate concepts devoted exclusively to linguistic content (i.e., Chomsky, Pinker), mentalistic content (i.e., Leslie, Baron-Cohen), and biological content (i.e., Medin, Atran). Domaingeneral mechanisms, on the other hand, are content-promiscuous. Presumably, deductive reasoning (as well as inductive, associative, and practical reasoning) operates roughly the same whether one is reasoning about tigers, lemons, or planets (but see Cosmides 1989). Now, a primary argument that nativists provide for their position is negative. They insist that domain-general learning mechanisms cannot adequately explain various epistemic competencies. This argument comes in both empirical and conceptual varieties. The former reasons from the purported failure of cognitive scientists to describe the relevant learning mechanisms. The later (see Fodor 1975, 1981) provides reasons for why domain-general explanations of conceptual change are in some sense incoherent.

The dynamics of analogical learning suggest empiricist responses to these negative arguments. Analogies take place across domains with different semantic content: comparing batteries to reservoirs, butchers to surgeons, or ideas to plants requires a flexible mechanism not devoted to any particular domain (though as we saw with progressive alignment, analogical learning is not restricted to cross-domain comparison). Thus, analogical cognition is a domain-general learning mechanism par excellence. In fact, a central appeal of Gentner's seminal 1983 paper was its elegant, syntactical description of analogical processes.

In response to the negative empirical argument, empiricists can note that nativists have focused on association, conditioning, and traditional empiricist accounts of abstraction while mostly neglecting comparison-based analogical learning. Consider the argument from the poverty of the stimulus, which is a common version of the negative argument. This argument claims that environmental input, together with the processing principles of 
a given general learning mechanism, is insufficient to explain some epistemic competency. However, what counts as a learning stimulus will depend on the candidate general-learning mechanism. We have to consider, then, the learning stimuli for analogical processes. According to structure-mapping theory, experience prompts analogical learning by culturally and naturally 'inviting' comparison (Gentner et al. 1995). Cultural invitations can occur through common labels or expressions that symbolically juxtapose objects or events, thereby invoking comparison. For example, labeling different cutting devices a "blick" will invite children to perform comparisons that result in the abstraction of a relational category (Gentner 2005). Natural invitations occur through spatio-temporal juxtapositions (compresent or contiguous objects/events), for example a child's repetitive act of knocking-over and reassembling a block tower (Gentner 2005).

Some recent explanations of the development of both syntactic knowledge and folk-psychological knowledge demonstrate how comparison opportunities, together with analogical abstraction and re-representation, deliver empiricist responses to the negative argument. Tomasello (2000) claims that children's syntactic competence can be explained by a usage account that relies on comparison-based analogical learning rather than an innate universal grammar. According to Tomasello's (1992) 'verb-island' hypothesis, young children's grasp of language is initially structured around specific verbs ('hit', 'broke') rather than general syntactic categories ('tense', 'object', 'agent'). His suggestion is that children can acquire these abstract categories by comparing concrete linguistic structures: '[Analogical learning] is exactly the kind of cognitive ability needed for children to create a verb island schema across different arrangements of object participants' (Tomasello 2000: 242). Another line of support for this approach comes from experiments and computational modeling of infant rule-learning. Marcus et al. (1999) report that 7-month-old infants who were exposed to a series of three-word artificial language sentences with the same grammatical pattern (e.g., ABA) were able to detect when new sentences violated the pattern. According to a structure-mapping analysis, the infants progressively aligned the grammatical structure through comparisons during the habituation trial. Kuehne et al. (2000) have used SEQL to computationally model this result as an example of learning through progressive alignment.

In the debate on the nature of folk-psychology, many empiricists are 'theory-theorists' who claim that children employ theory-building skills (they are 'little scientists') in order to build psychological theories that are predictive and explanatory of intentional behavior. Acknowledging the force of the negative empirical argument, theory-theorists such as Gopnik and Schulz note:

It is all very well to suggest that children's learning mechanisms are analogous to scientific theoryformation. However, what we would really like is a more precise specification of the mechanisms that underlie learning in both scientists and children (Gopnik and Schulz 2004: 371).

Gopnik and Schulz enlist Bayesian causal graphical modeling as such a mechanism. Bach (2011) suggests an alternative. He claims that mental simulation and pretend role-play require the comparison of representations with psychological content. Given the ampliative principles of structure-mapping, this suggests that simulation and role-play will facilitate the abstraction of theoretical schemas - schemas that describe cognitive-behavioral relations. In other words, simulation - construed as a type of structural comparison - produces psychological theory. Furthermore, exposure to sentential complements ('The doctor says that the apples are healthy') and contrastive conversations (see Bartsch and Wellman 1995) provide cultural invitations for the structural comparison of representations with psychological-behavioral content. The implication is that young 
folk-psychologists may not need theory-of-mind modules. Instead, experience provides comparison opportunities that allow children to progressively align cognitive-behavioral relations that describe the essential features of folk-psychological categories such as belief, desire and the belief-desire law.

Supporters of conceptual arguments against general-learning are unlikely to be swayed by the above types of considerations. Perhaps, though, they might qualify the implications they credit to their argument. I cannot do justice to Fodor's argument here (see Cowie 1999; Laurence and Margolis 2002), but a key premise is that rational learning occurs through a process of hypothesis formation and testing. This is noteworthy because learning through analogy does not require hypothesis formation and testing. For example, children's' comparison-based abstractions as reported in Kotovsky and Gentner (1996) did not involve experimenter feedback or hypothesis revision. Of course, analogical learning does not occur ex nihilo - there must first be representations to align. Nonetheless, the learner does not construct hypotheses - explicitly or implicitly - as to the types of representational structures that emerge from alignment. (On the other hand, the general learning mechanism of Bayesian causal inference does require hypothesis formation - see especially Perfors et al. 2011, section 5.3.; Christie and Gentner 2010.) See also Gentner 2003, section 8.6.4.

Fodor is likely to dig in here, insisting that 'rational' learning requires experiences that bear a 'confirmation relation' to hypotheses, and that for this reason analogy-learning is 'brute-causal' rather than rational. Can empiricists challenge this stipulation? Perhaps they can appeal to the case of scientific pioneers such as Kepler and Einstein (exemplars of learning and rationality) for whom analogy played a central role in the discovery process. Relatedly, they can reference Dunbar's 'in vivo' empirical studies of live science which report that 'analogical thinking is a key component of all aspects of scientific reasoning, ranging from hypothesis generation to experimental design, data interpretation, and explanations' (Dunbar 2001: 315). If empiricists prefer to concede Fodor's conceptual point, they might still point out that comparison-based abstraction makes available an alternative compositional route (but not in Fodor's sense) from simple concepts to complex concepts. The availability of such a route - even if it is brute casual rather than rational - provides one possible explanation of how concepts such as carburetor and telecommunication can arise from simpler concepts rather than from innate endowment. ${ }^{1}$

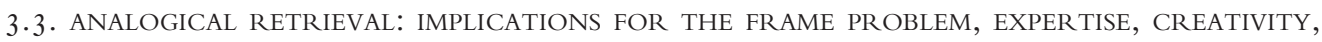 AND AUTISM}

Very roughly, the frame problem concerns how relevant information is brought to bear on a given situation or task. We know that our brains can sort the relevant from the irrelevant - the mystery is how they do so in a computationally feasible way. As Fodor (1983) points out, the frame problem is front and center for unencapsulated mechanisms like analogy because, having access to content from any domain, any content is potentially relevant. But the issue is more precise for analogy because relevancy is a concern specifically for analogical retrieval. Suppose that you are reading a magazine article that describes the ways in which a particular materialistic society has discouraged the artistic ambitions of a recent college graduate. This article could invoke in you any number of potential base representations stored in semantic memory. Perhaps you are reminded of an artist friend of yours. Or, to borrow a description from Mill, you are reminded of 'a very tender plant, easily killed, not only by hostile influences, but by mere want of 
sustenance' (Mill 1871: 15). Which of these representations, or any of the countless others, will be activated and subsequently aligned with your representation of the graduate?

Empirical studies of retrieval suggest a partial answer. Unfortunately, the news is not encouraging for our epistemic and problem-solving goals. While analogical mapping is driven by relational similarity, retrieval tends to be driven by superficial and literal similarity (Gick and Holyoak 1980, 1983; Ross 1987; Gentner et al. 1993). In other words, people tend to access representations that look like, or have the same surface features as, target problems and representations. This retrieval method is epistemically adequate if the (superficially similar) base representation shares causal and relational properties with the target. But obviously this is not always the case: things that look like predators can be friendly and harmless, and the cute and cuddly things turn out to be vicious killers. Given the explanatory and predictive value of relational and causal properties (and thus relational concepts and schemas), it is less than ideal that our retrieval bias often leaves the most explanatory analogues dormant in semantic memory. There is some hope, however, if you are an expert, in which case you have considerable domain knowledge. Experts, because they better encode knowledge representations with explicit relations, are more apt to retrieve relationally similar base analogues (Novick 1988; Gentner et al. 2003).

These considerations may be part of an answer to the Frame Problem, but more needs to be said about which of the superficially similar representations, or which of the relationally similar representations, are activated as base analogues. Along these lines, Shanahan and Baars (2005) offer an interesting proposal for how massive parallel processing, where representations 'compete' for activation in a global work space, might combine with computational theories of analogy to provide a rich solution to the Frame Problem.

Finally, it is worth mentioning that an adequate theory of analogical retrieval could shed light on, or offer possible reductions of, the notions of creativity and generativity (see Turner 1997; Jarrold 1997 for discussion of generativity). This is because generativity tasks and many paradigm cases of creativity involve the transfer of relational roles and relational systems to novel domains. Construing generativity skill as a form of analogical retrieval may also help clarify the relationship between analogical cognition and autism. Morsanyi and Holyoak's (2010) study shows intact analogical transfer ability for autistic individuals, but because the tasks in this study supplied subjects base analogues, the study does not tell us about retrieval ability in autism. Given that autism appears to correlate with impaired generativity (Jarrold 1997; Peterson and Bowler 2000), future research might investigate a connection between autism and impaired relational retrieval. (Note also that, given the considerations advanced in Section 3.2. about the developmental relationship between analogy and folk-psychology, impaired analogical retrieval may also be explanatory of theory-of-mind deficit. As such, an analogy-based explanation of autism could have considerable parsimony.)

\subsection{COGNITIVE ARCHITECTURE}

One way to support your favorite theory of cognitive architecture is to isolate some nonnegotiable feature of cognition - the systematicity of thought, for example - and then argue that only your preferred cognitive architecture can explain it. Is analogy a nonnegotiable feature of cognition? Hofstadter believes so:

If analogy were merely a special variety of something that in itself lies way out on the peripheries, then it would be but an itty-bitty blip in the broad blue sky of cognition. To me, however, 
analogy is anything but a bitty blip - rather, it's the very blue that fills the whole sky of cognition - analogy is everything, or very nearly so, in my view. (Hofstadter 2001: 499)

Gentner (2003) and Penn et al. (2008) express similar views. It is becoming more widely accepted, however, that not all architectures are currently equal in their explanation of analogical cognitive abilities. Gentner reports that 'structured symbolic models, such as SME ... and structured symbolic-connectionist models, such as LISA ... have had the greatest success in accounting for the range of phenomena in analogical thinking and learning' (Gentner 2010: 336). On the other hand, non-symbolic and sub-symbolic architectures, such as those posited in dynamic systems theory and distributed connectionism, have not enjoyed the same success. The reason given for this disparity is that structured symbolic representations have the flexibility to express the relational roles and relational systems that are at the core of analogical cognition. (See also Markman 1999; Holyoak and Hummel 2000; Markman and Gentner 2000; French 2008. For defenses of subsymbolic approaches to analogy see Eliasmith and Thagard 2001; Leech et al. 2008.)

\subsection{RELATIONAL KNOWLEDGE AND THINKING}

Theories of analogy have paved the way for a number of recent articles that discuss the importance of 'role-governed categories' (Markman and Stillwell 2001), 'relational categories' (Gentner 2005; Gentner and Kurtz 2005), and 'relational thinking' (Doumas et al. 2008; Halford et al. 2010). The type of conceptual content explored in these articles bears interesting similarities to the types of mental states described in the philosophical literature on psycho-functionalism and theory-based concepts. One might view recent work on relational categories as offering an empirically informed elaboration on the structure and dynamics of conceptual role (Block 1986). On the other hand, it is not clear whether, or how, relational categories (and more generally theories of analogy) bear on the referential aspects of conceptual content.

There is now growing consensus that relational knowledge and the capacity for higher-order, relational cognition are at the core of many signature human cognitive abilities (Gentner 2003; Halford et al. 2010). There is also growing consensus that the ability for higher-order relational thought and abstraction - an ability that is constitutive of and facilitated by analogical cognition - is not possessed by other species to any interesting degree, even chimpanzees (Penn et al. 2008). While most animals have some capacity for learning by association and conditioning, the ability to form relational abstractions and reason analogically may be a defining and unique feature of human cognition.

\section{Conclusion}

It was once customary to regard analogical processes as those occasional instances in which one thinks of a situation in terms of some other situation. Contrary to this, cognitive scientists now understand analogical cognition as a ubiquitous cognitive process that occurs even during mundane comparison. Moreover, there is now good evidence that analogical ability is central to the human capacity for relational thinking, epistemic discovery, and creativity. Philosophers investigating the nature of mind, language, and knowledge would do well to grapple with these new and exciting advances. I hope this article is instrumental to that end. 


\section{Short Biography}

Theodore Bach received his $\mathrm{PhD}$ from the University of Connecticut in August 2010. $\mathrm{He}$ is currently assistant professor of philosophy at Bowling Green State University Firelands College. His research focuses on several topics in the philosophy of psychology including analogical cognition, mental simulation, and pretense. He also researches the status of natural kinds, particularly with respect to the social sciences. Recent publications include "Structure-Mapping: Directions from Simulation to Theory" in Philosophical Psychology, and "Gender is a Natural Kind with a Historical Essence," forthcoming in Ethics.

\section{Notes}

* Correspondence: One University Drive, Huron, OH 44074, USA. Email: theodorebach@gmail.com.

${ }^{1}$ See also Lakoff and Johnson (1999) for a discussion of how primary metaphors, which recruit sensorimotor inferential structure, enable grasp of abstract concepts.

\section{Works Cited}

Bach, T. 'Structure-Mapping: Directions from Simulation to Theory.' Philosophical Psychology 24.1 (2011): 23-51.

Baron-Cohen, S. Mindblindness: An Essay on Autism and Theory of Mind. Cambridge, MA: MIT Press, 1995.

Bartsch, K. and H. M. Wellman. Children Talk About the Mind. New York: Oxford University Press, 1995.

Block, N. 'Advertisement for a Semantics for Psychology.' Midwest Studies in Philosophy, Volume X: Studies in the Philosophy of Mind. Eds. P. A. French, T. Uehling Jr., and H. Wettstein. Minneapolis: University of Minnesota Press, 1986. 615-78.

Chalmers, D. 'Facing Up to the Problem of Consciousness.' Journal of Consciousness Studies 2.3 (1995): 200-19.

Christie, S. and D. Gentner. 'Where Hypotheses Come From: Learning New Relations by Structural-Alignment.' Journal of Cognition and Development 11.3 (2010): 356-73.

Cosmides, L. 'The Logic of Social Exchange: Has Natural Selection Shaped How Humans Reason? Studies with the Wason Selection Task.' Cognition 31 (1989): 187-276.

Cowie, F. What's Within? Nativism Reconsidered. New York: Oxford University Press, 1999.

Davies, M. 'Knowledge (Explicit and Implicit): Philosophical Aspects.' International Encyclopedia of the Social and Behavioral Sciences. Eds. N. J. Smelser, and P. B. Baltes. Amsterdam: Elsevier Science Ltd., 12, 2001. $8126-32$.

- and T. Stone. 'Mental Simulation, Tacit Theory, and the Threat of Collapse.' Philosophical Topics 29.1/2(2001): 127-73.

Dienes, Z. and J. Perner. 'A Theory of Implicit and Explicit Knowledge.' Behavioral and Brain Sciences 22 (1999): 735-808.

Doumas, L., J. Hummel and C. A. Sandhofer. 'Theory of the Discovery and Predication of Relational Concepts.' Psychological Review 115.1 (2008): 1-43.

Dunbar, K. 'The Analogical Paradox: Why Analogy is So Easy in Naturalistic Settings Yet So Difficult in the Psychological Laboratory.' The Analogical Mind: Perspectives from Cognitive Science. Eds. D. Gentner, K. J. Holyoak, and B. N. Kokinov. Cambridge, MA: MIT Press, 2001. 313-34.

Eliasmith, C. and P. Thagard. 'Integrating Structure and Meaning: A Distributed Model of Analogical Mapping.' Cognitive Science 25 (2001): 245-86.

Fodor, J. The Language of Thought. Cambridge, MA: Harvard University Press, 1975.

- Modularity of Mind. Cambridge, MA: MIT Press, 1983.

- 'The Present Status of the Innateness Controversy.' Representations: Philosophical Essays on the Foundations of Cognitive Science. Cambridge, MA: MIT Press, 1981.

Forbus, Kenneth D., et al. 'Analogy Just Looks Like High Level Perception: Why a Domain General Approach to Analogical Mapping is Right.' Journal of Experimental and Theoretical Artificial Intelligence 10 (1998): 231-57.

French, R. M. 'Relational Priming is to Analogy-Making as One-Ball Juggling is to Seven-Ball Juggling.' Behavioral and Brain Sciences 31 (2008): 386-7.

Gentner, D. 'The Development of Relational Category Knowledge.' Building Object Categories in Developmental Time. Eds. L. Gershkoff-Stowe, and D. H. Rakison. Hillsdale, NJ: Erlbaum, 2005. 245-75.

—. 'Psychology in Cognitive Science: 1978-2038.' Topics in Cognitive Science 2 (2010): 328-44.

. 'Structure-Mapping: A Theoretical Framework for Analogy.' Cognitive Science 7 (1983): 155-70. 
'Why We're So Smart.' Language in Mind: Advances in the Study of Language and Thought. Eds. D. Gentner, and S. Goldin-Meadow. Cambridge, MA: MIT Press, 2003. 195-235.

— and J. Colhoun. 'Analogical Processes in Human Thinking and Learning.' On Thinking: Vol. 2. Towards a Theory of Thinking. Series Eds. A. von Müller, and E. Pöppel, Vol. Eds. B. Glatzeder, and V. Goel, A. von Müller. Berlin Heidelberg: Springer-Verlag, 2010. 35-48.

- and K. Forbus. 'Computational Models of Analogy.' WIREs Cognitive Science 2 (2011): 266-76.

and K. Kurtz. 'Relational Categories.' Categorization Inside and Outside the Lab. Eds. W. K. Ahn, R. L. Goldstone, B. C. Love, A. B. Markman, and P. W. Wolff. Washington, DC: APA, 2005. 151-75.

and —. 'Relations, Objects, and the Compositions of Analogies.' Cognitive Science 30 (2006): 609-42.

and A. Markman. 'Structure-Mapping in Analogy and Similarity.' American Psychologist 52.1 (1997): 45-56.

and J. Medina. 'Similarity and the Development of Rules.' Cognition 65 (1998): 263-97.

, J. Loewenstein and L. Thompson. 'Learning and Transfer: A General Role for Analogical Encoding.' Journal of Educational Psychology 95 (2003): 393-408.

- M. J. Rattermann and K. D. Forbus. 'The Roles of Similarity in Transfer: Separating Retrievability from Inferential Soundness.' Cognitive Psychology 25 (1993): 524-75.

- , et al. 'Analogy in Discovery: A Case Study of Johannes Kepler.' Creative Thought: An Investigation of Conceptual Structures and Processes. Eds. T. B. Ward, S. M. Smith, and J. Vaid. Washington, DC: American Psychological Association, 1997. 403-59.

— Approaches to Process Modeling. Eds. T. J. Simon, and G. S. Halford. Hillsdale, NJ: Lawrence Erlbaum Associates, Inc., 1995. 263-313.

Gick, M. L. and K. J. Holyoak. 'Analogical Problem Solving.' Cognitive Psychology 12 (1980): 306-55.

$\longrightarrow$ and —. 'Schema Induction and Analogical Transfer.' Cognitive Psychology 15 (1983): 1-38.

Gopnik, A. and L. E. Schulz. 'Mechanisms of Theory-Formation in Young Children.' Trends in Cognitive Science 8 (2004): 371-7.

Halford, G. S., W. H. Wilson and S. Phillips. 'Relational Knowledge: The Foundation of Higher Cognition.' Trends in Cognitive Science 14.11 (2010): 497-505.

Hesse, M. B. Models and Analogies in Science. Notre Dame, IN: University of Notre Dame Press, 1966.

Hofstadter, D. 'Epilogue: Analogy as the Core of Cognition.' The Analogical Mind: Perspectives from Cognitive Science. Eds. D. Gentner, K. J. Holyoak, and B. N. Kokinov. Cambridge, MA: MIT Press, 2001. 499-538.

Holyoak, K. J. and J. E. Hummel. 'The Proper Treatment of Symbols in a Connectionist Architecture.' Cognitive Dynamics: Conceptual and Representational Change in Humans and Machines. Eds. E. Dietrich, and A. Markman. Mahwah, NJ: Lawrence Erlbaum Associates, 2000. 229-63.

— and - 'Towards an Understanding of Analogy within a Biological Symbol System.' The Analogical Mind: Perspectives from Cognitive Science. Eds. D. Gentner, K. J. Holyoak, and B. N. Kokinov. Cambridge, MA: MIT Press, 2001. 161-95.

Jarrold, C. 'Pretend Play in Autism: Executive Explanations.' Autism as an Executive Disorder. Ed. J. Russell. New York: Oxford University Press, 1997, 101-40.

Kotovsky, L. and D. Gentner. 'Comparison and Categorization in the Development of Relational Similarity.' Child Development 67 (1996): 2797-822.

Kuehne, S. E., et al. 'SEQL - Category Learning as Incremental Abstraction Using Structure Mapping.' Proceedings of the Twenty second meeting of the Cognitive Science Society. August 13-15, 2000, Institute for Research in Cognitive Science, University of Pennsylvania: Philadelphia, PA. Hillsdale, NJ: Lawrence Erlbaum Associatesin, 2000.

Lakoff, G. and M. Johnson. Philosophy in the Flesh: The Embodied Mind and Its Challenge to Western Thought. New York: Basic Books, 1999.

Laurence, S. and E. Margolis. 'Radical Concept Nativism.' Cognition 86 (2002): 22-55.

Leech, R., D. Mareschal and R. P. Cooper. 'Analogy as Relational Priming: A Developmental and Computational Perspective on the Origins of Complex Cognitive Skill.' Behavioral and Brain Sciences 31 (2008): 357-414.

Marcus, G. F., et al. 'Rule-Learning in Seven-Month-Old Infants.' Science 283 (1999): 77-80.

Markman, A. B. Knowledge Representation. Mahwah, NJ: Lawrence Erlbaum Associates, 1999.

— and D. Gentner. 'Structure-Mapping in the Comparison Process.' Journal of Psychology 113.4 (2000): 50138.

— and C. H. Stillwell. 'Role-Governed Categories.' Journal of Experimental and Theoretical Artificial Intelligence 13.4 (2001): 329-58.

Mill, J. S. Utilitarianism. London: Longmans, Green, Reader, and Dyer, 1871.

Morsanyi, K. and K. Holyoak. 'Analogical Reasoning Ability in Autistic and Typically Developing Children.' Developmental Science 13.4 (2010): 578-87.

Novick, L. R. 'Analogical Transfer, Problem Similarity, and Expertise'' Journal of Experimental Psychology: learning, Memory, and Cognition 14 (1988): 510-20.

Penn, D. C., K. J. Holyoak and D. J. Povinelli. 'Darwin's Mistake: Explaining the Discontinuity between Human and Nonhuman Minds.' Brain and Behavioral Sciences 31 (2008): 109-78. 
Perfors, A., et al. 'A Tutorial Introduction to Bayesian Models of Cognitive Development.' Cognition 120 (2011): 302-21.

Peterson, D. and D. M. Bowler. 'Counterfactual Reasoning and False Belief Understanding in Children with Autism.’ Autism 4.4 (2000): 391-405.

Ross, B. H. 'This is Like That: The Use of Earlier Problems and the Separation of Similarity Effects.' Journal of Experimental Psychology: learning, Memory, and Cognition 13.4 (1987): 629-39.

Shanahan, M. and B. Baars. 'Applying Global Workspace Theory to the Frame Problem.' Cognition 98 (2005): 157-76.

Stich, S. and S. Nichols. 'Folk Psychology: Simulation or tacit theory?' Mind and Language 7 (1992): 35-71.

Tomasello, M. 'Do Young Children Have Adult Syntactic Competence?' Cognition 74 (2000): 209-53.

- First Verbs. New York: Cambridge University Press, 1992.

Turner, M. A. 'Towards and Executive Dysfunction Account of Repetitive Behavior in Autism.' Autism as an Executive Disorder. Ed. J. Russell. New York: Oxford University Press, 1997, 57-100. 